\title{
LAMB3 mediates apoptotic, proliferative, invasive, and metastatic behaviors in pancreatic cancer by regulating the $\mathrm{Pl} 3 \mathrm{~K} /$ Akt signaling pathway
}

\author{
Hong Zhang ${ }^{1,2,3,4}$, Yao-zhen Pan ${ }^{2,3}$, May Cheung ${ }^{4}$, Mary Cao ${ }^{4}$, Chao Yu ${ }^{2,3}$, Ling Chen ${ }^{2,3}$, Lei Zhan ${ }^{2,3}$, Zhi-wei He ${ }^{2,3}$ and \\ Cheng-yi Sun ${ }^{2,3}$
}

\begin{abstract}
The poor prognosis of patients with pancreatic ductal adenocarcinoma (PDAC) is partially attributed to the invasive and metastatic behavior of this disease. Laminin subunit beta-3 (LAMB3) encodes one of the three subunits of LM-332, an extracellular matrix protein secreted by cultured human keratinocytes. In addition, LAMB3 is involved in the invasive and metastatic abilities of some types of cancer, including colon, pancreas, lung, cervix, stomach, and prostate cancer, but the role and mechanism of LAMB3 in PDAC have not been previously determined. Herein, we tentatively investigated the role of LAMB3 in the malignant biological behavior of PDAC. In this study, we demonstrated that LAMB3 is upregulated in PDAC. Inhibition of LAMB3 abrogated the tumorigenic outcomes of PI3K/Akt signaling pathway activation, including those involving cell cycle arrest, cell apoptosis, proliferation, invasion and migration in vitro, and tumor growth and liver metastasis in vivo. Our results showed that LAMB3 could mediate cell cycle arrest and apoptosis in PDAC cells and alter the proliferative, invasive, and metastatic behaviors of PDAC by regulating the PI3K/Akt signaling pathway. LAMB3 may be a novel therapeutic target for the treatment of PDAC in the future.
\end{abstract}

\section{Introduction}

Pancreatic ductal adenocarcinoma (PDAC) is a malignant tumor that is difficult to diagnose early and treat worldwide $^{1}$. Despite improvements in treatment, patient prognosis remains poor, partly attributed to devastating local tumor invasion and distant metastasis ${ }^{2,3}$. PDAC develops as a result of genetic and epigenetic alterations. Therefore, obtaining a better understanding of the potential mechanisms for the growth, metastasis, apoptosis, and tumorigenic properties of PDAC will provide opportunities for the development of new therapeutic

\footnotetext{
Correspondence: Cheng-yi Sun (sunchengyi2014@163.com)

${ }^{1}$ Guizhou Medical University, Guiyang, Guizhou, China

${ }^{2}$ Department of Hepatobiliary Surgery, The Affiliated Hospital of Guizhou

Medical University, Guiyang, Guizhou, China

Full list of author information is available at the end of the article.

Edited by A. Oberst
}

strategies for this disease $\mathrm{e}^{4,5}$. We analyzed expression profiles in The Cancer Genome Atlas (TCGA) and Gene Expression Omnibus (GEO) databases and found laminin subunit beta-3 (LAMB3) to be upregulated in PDAC . We $^{6}$ investigated LAMB3 expression in PDAC using 102 matched pairs of PDAC and adjacent normal pancreatic tissues with a tissue microarray (TMA) and found that the product of this gene, which encodes a member of the kinesin protein family, may play a vital role in PDAC carcinogenesis.

Laminins are important and biologically active components of the basal lamina that influence cell differentiation, migration, adhesion, proliferation, and survival ${ }^{7,8}$. LAMB3 encodes one of the three subunits of LM-332, an extracellular matrix protein secreted by cultured human keratinocytes. While LAMB3 is involved in the invasive and metastatic abilities of some types of cancer, including

\section{(c) The Author(s) 2019}

(c) (i) Open Access This article is licensed under a Creative Commons Attribution 4.0 International License, which permits use, sharing, adaptation, distribution and reproduction cc) in any medium or format, as long as you give appropriate credit to the original author(s) and the source, provide a link to the Creative Commons license, and indicate if changes were made. The images or other third party material in this article are included in the article's Creative Commons license, unless indicated otherwise in a credit line to the material. If material is not included in the article's Creative Commons license and your intended use is not permitted by statutory regulation or exceeds the permitted use, you will need to obtain permission directly from the copyright holder. To view a copy of this license, visit http://creativecommons.org/licenses/by/4.0/. 
colon, pancreas, lung, cervix, stomach, and prostate cancer, its mechanism of action in pancreatic cancer has not been investigated previously ${ }^{9-11}$.

Phosphatidylinositol 3-kinase (PI3K) and protein kinase $\mathrm{B}$ (PKB/Akt) are the key proteins in the PI3K/Akt signaling pathway. This pathway is regulated by multiple mechanisms and is involved in numerous types of cancer $^{12-14}$. Activated Akt is involved in regulating cell cycle and the proliferative, antiapoptotic, metastatic, and invasive abilities of cancer cells ${ }^{15-17}$. Phosphoinositide-dependent kinase 1 (PDK1) partially activates Akt via phosphorylation of T308, and phosphorylation of S473 by PDK2 is needed for full activation ${ }^{18,19}$.

Our results show that LAMB3 is upregulated in PDAC, and suppressing its expression reduces cell proliferation, invasion, and migration by downregulating epithelialmesenchymal transition (EMT)-related proteins (N-cadherin, vimentin, Snail, Slug). LAMB3 suppression also significantly decreases Akt phosphorylation and inhibits the transcription of PI3K, reducing its activation. These results suggest that LAMB3 promotes tumor invasion via Akt activation through the PI3K axis in PDAC cells. Our findings identified a novel molecular mechanism of action for LAMB3 in PDAC, potentially suggesting a novel therapeutic strategy for blocking PDAC invasion and metastasis.

\section{Results}

LAMB3 is positively correlated with PDAC development

Through analyzing published messenger RNA (mRNA) expression profiles in the NCBI GEO (GSE35141; https:// www.ncbi.nlm.nih.gov/geo/), we found that LAMB3 mRNA was significantly upregulated in PDAC tissues compared with normal pancreas tissues. The gene set enrichment analysis (GSEA) plot indicated that high LAMB3 expression was significantly positively correlated with PDAC adhesion and migration by activating the phosphatidylinositol signaling system (Fig. 1a). The TCGA dataset showed that LAMB3 upregulation was associated with PDAC disease stage (Fig. 1b). The heat map showed the relative median expression of LAMB3 and genes positively correlated with LAMB3 in eight common solid tumors, PAAD (pancreatic adenocarcinoma), COAD (colon adenocarcinoma), LIHC (liver hepatocellular carcinoma), PRAD (prostate adenocarcinoma), LUAD (lung adenocarcinoma), THCA (thyroid carcinoma), BRCA (invasive breast carcinoma), and BLCA (bladder urothelial carcinoma), based on datasets from the TCGA database; LAMB3 was positively correlated with PDAC (Fig. 1c).

\section{LAMB3 expression is increased in PDAC tissues}

We analyzed LAMB3 mRNA expression levels in 20 paired patient samples of PDAC and adjacent normal tissues. LAMB3 expression was obviously increased in
PDAC tissues compared with normal tissues (Fig. 2a). We confirmed this finding through TMAs containing 102 matched pairs of PDAC and adjacent normal pancreas tissues (Fig. 2b). According to the total immunohistochemistry (IHC) score (percentage of positive cells $\mathrm{x}$ staining intensity), LAMB3 was expressed at higher levels in PDAC tumor tissues than in adjacent normal tissues (Fig. 2c). Moreover, an analysis of the clinical characteristics of PDAC revealed that high LAMB3 expression was related to more advanced tumor node metastasis (TNM) stage (Supplementary Table 1). Furthermore, Kaplan-Meier survival curves revealed an obvious correlation between high LAMB3 expression and poor prognosis of patients with PDAC ( $P=0.0026$; Fig. $2 \mathrm{~d})$.

\section{LAMB3 is expressed in PDAC cells and promotes PDAC cell proliferation in vitro}

We investigated LAMB3 mRNA levels in seven PDAC cell lines and human pancreatic ductal epithelial (HPDE) cells (Fig. 3a). LAMB3 expression levels were upregulated in the seven PDAC cell lines compared with the HPDE cells. Then, we chose three cell lines with the highest LAMB3 protein expression levels to evaluate LAMB3 expression after cell manipulation (Fig. 3c). LAMB3 expression was obviously increased in PANC- 1 and MIA $\mathrm{PaCa}-2$ cells at both the mRNA and protein levels. We also examined LAMA3 and LAMC2 protein expression and found it to be increased in the PDAC cell lines compared to the normal pancreatic cell line. We used a lentiviral delivery system to generate MIA PaCa-2 cells and PANC-1 cells stably overexpressing LAMB3 (LAMB3U) or with stable LAMB3 knockdown (LAMB3D). To confirm LAMB3 overexpression and silencing, we compared LAMB3U and LAMB3D cells with the corresponding negative control (NC) cells by quantitative PCR (qPCR) and western blotting. GAPDH was used as the loading control (Fig. 3b-d). Cell counting kit-8 (CCK-8) and colony formation assays suggested that PDAC proliferation was inhibited by LAMB3 knockdown and increased by LAMB3 overexpression (Fig. 3e).

\section{LAMB3 promotes PDAC cell migration and invasion in vitro}

As our results indicated that LAMB3 expression correlated with local invasion and metastasis, we investigated the effect of LAMB3 on the invasive and metastatic capacities of PDAC cells using transwell and wound healing assays. We evaluated the wound width at $0,24,48$, and $72 \mathrm{~h}$ after virus infection in wound healing assays and the percentage of invasive and metastatic cells at $24 \mathrm{~h}$ after virus infection in transwell assays. Transwell and wound healing assays both revealed that PDAC cell invasion and migration in vitro was promoted by LAMB3 overexpression and inhibited by LAMB3 knockdown (Fig. 4a, b). 


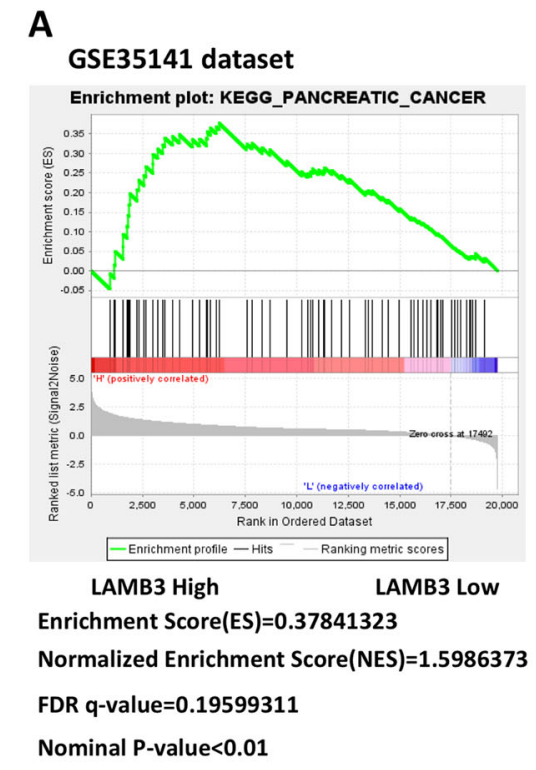

A

B

TCGA dataset

Expression of LAMB3 in PDAC base on major cancer stages

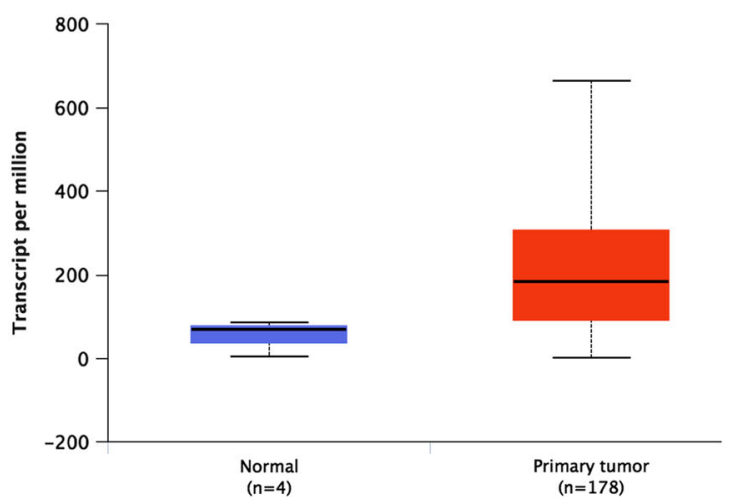

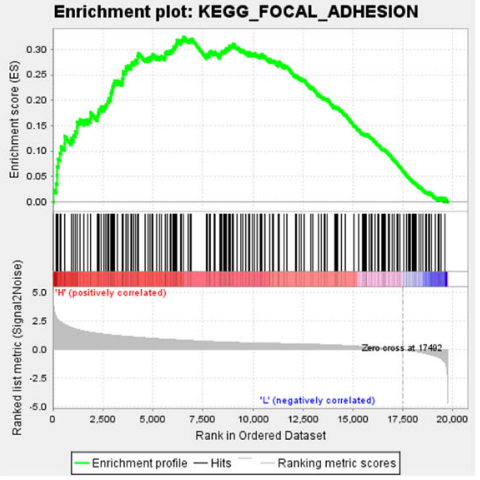

LAMB3 High Enrichment Score(ES) $=0.324607$

Normalized Enrichment Score(NES) $=1.5187625$ FDR q-value $=0.25397405$

Nominal P-value $<0.01$

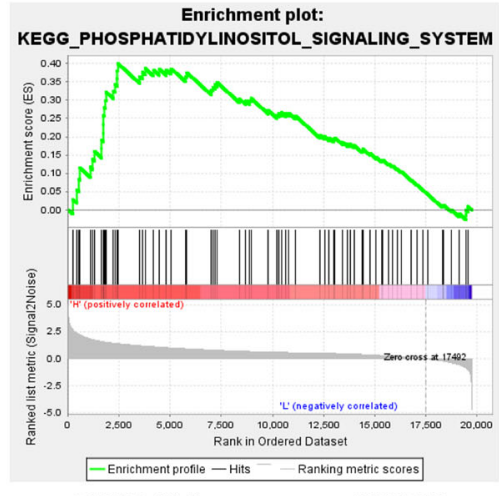

LAMB3 High

Enrichment Score(ES) $=0.4009782$

Normalized Enrichment Score(NES)=1.7996309

FDR q-value $=0.16331193$

Nominal P-value $<0.01$

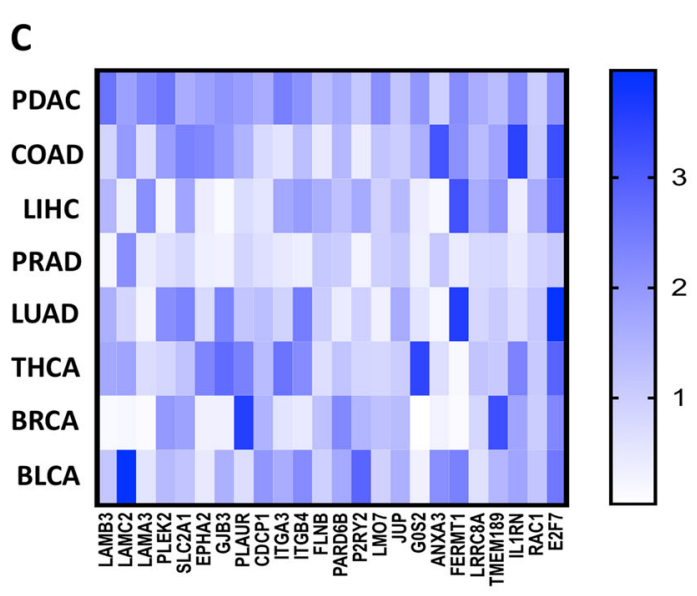

Fig. 1 LAMB3 is positively correlated with pancreatic ductal adenocarcinoma (PDAC) development. a The gene set enrichment analysis (GSEA) plot indicated that high LAMB3 expression was significantly positively correlated with PDAC. b The Cancer Genome Atlas (TCGA) dataset suggested that LAMB3 was correlated with PDAC disease stage. $\mathbf{c}$ Heat map showing the relative expression of LAMB3 and genes positively correlated with LAMB3 in eight common solid tumors

LAMB3 affects apoptosis and the cell cycle distribution of PDAC cells in vitro

Flow cytometry was used to explore whether LAMB3 promotes PDAC proliferation by regulating the cell cycle and/or apoptosis. Changes in LAMB3 levels affected the apoptotic ratio. In both cell lines with LAMB3 knockdown, the population of apoptotic cells increased, especially that of cells in early apoptosis. In both cell lines overexpressing LAMB3, only the number of early apoptotic cells decreased (Fig. 5a). Moreover, LAMB3 induced a dramatic alteration in cell cycle distribution. In both cell lines with LAMB3 knockdown compared with NC cells, the fraction of cells in G1 phase increased, while that of cells in S and G2/M phase decreased. Moreover, in both cell lines overexpressing LAMB3 compared with NC cells, the fraction of cells in G1 phase decreased, while that of cells in G2/M phase increased (Fig. 5b). Western blot and qPCR analyses showed that LAMB3 overexpression upregulated cyclin D1, which promoted cell cycle progression, and BCL-2, which inhibited apoptosis, and downregulated $\mathrm{p} 53$, which arrested cell cycle progression and promoted apoptosis; the LAMB3 knockdown group had the opposite effect (Fig. 5c, d).

\section{LAMB3 regulates PI3K-mediated Akt phosphorylation and EMT in PDAC cell lines}

As mentioned above, our GSEA plot results indicated that high LAMB3 expression was significantly positively 


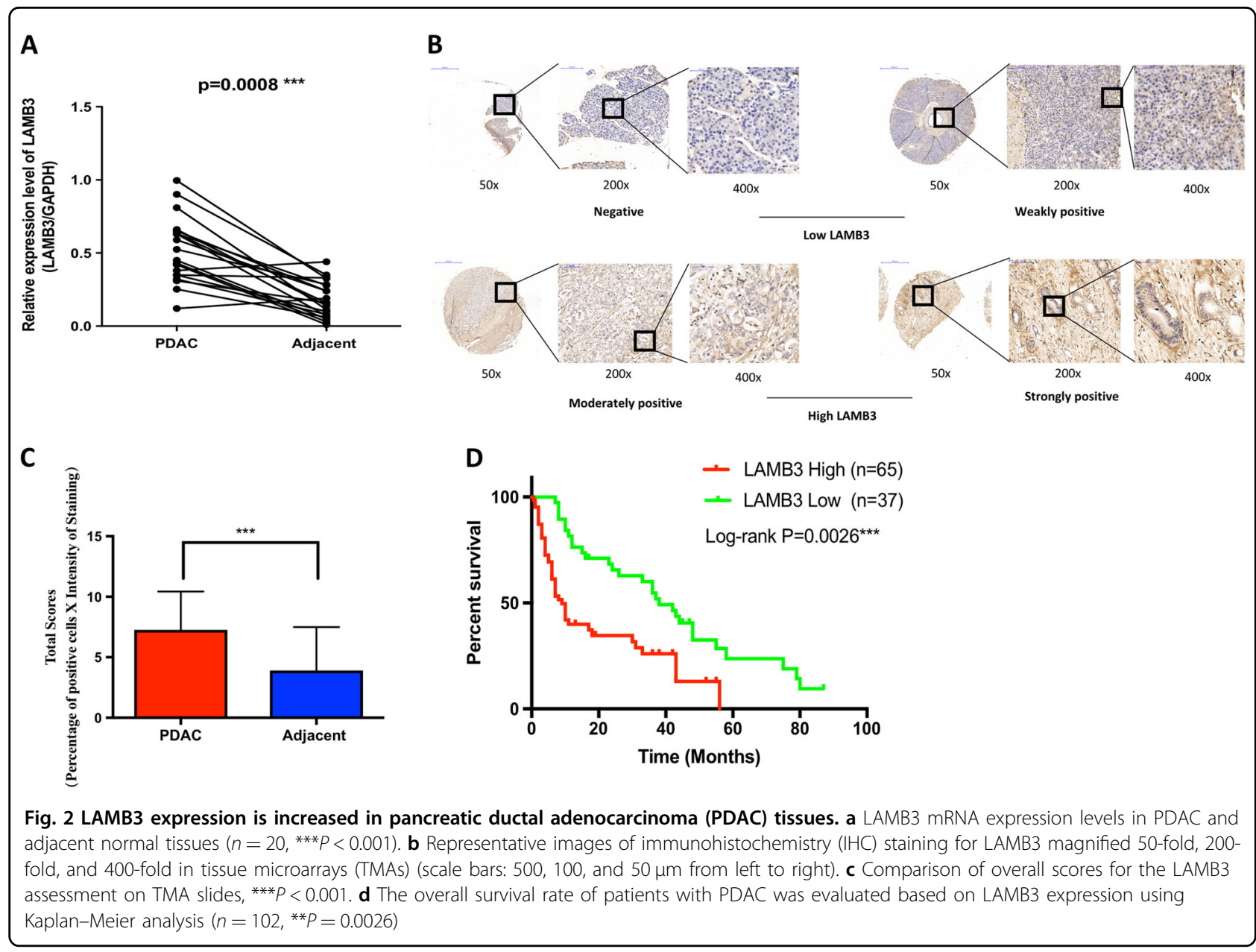

correlated with PDAC adhesion and migration via activation of the phosphatidylinositol signaling system. PI3K/ Akt signaling appears to play an important role in PDAC progression. Inhibiting Akt with LY294002 decreased the protein levels of the EMT-related proteins vimentin, Slug, and Snail, and increased those of the epithelial marker Ecadherin (Fig. 6a). The protein expression levels of the tumor invasion and migration-related proteins matrix metalloproteinase 9 (MMP9) and MMP2 were also reduced by Akt inhibition (Fig. 6b). To elucidate whether inhibiting the PI3K/Akt signaling pathway can reduce PDAC cell invasion and migration, transwell invasion, and migration assays were performed using LY294002-treated PANC-1 and MIA PaCa- 2 cells with or without LAMB3 overexpression. Inhibition of the PI3K/Akt signaling pathway significantly suppressed invasion and migration in both cell lines (Fig. 6c). Furthermore, we hypothesized that LAMB3 regulates cell migration and invasion via the PI3K/Akt signaling pathway. LAMB3 knockdown significantly decreased the levels of the EMT-related proteins Slug, Snail, vimentin, and N-cadherin, and increased E-cadherin levels; the levels of PI3K, PDK2, and phosphorylated Akt at S473 (p-Akt-S473) were also decreased, western blotting revealed that total Akt levels were not affected in either cell line, and LAMB3 overexpression had the opposite effect (Fig. 6d). We have shown that LAMB3 promotes PANC-1 and MIA PaCa-2 cell invasion and migration by transwell invasion and migration assays. To further verify whether these effects are mediated through the PI3K/Akt pathway at the protein level, NC and LAMB3-overexpressing PANC-1 and MIA PaCa-2 cells were treated with the Akt inhibitor LY294002, and the levels of Akt, p-Akt-S473 and the EMT-related proteins N-cadherin, E-cadherin, Snail, and Slug were examined by western blotting. LY294002 significantly decreased the levels of p-AktS473, N-cadherin, Snail, and Slug in both the NC and LAMB3-overexpressing groups, whereas E-cadherin levels were significantly increased in only LAMB3overexpressing MIA PaCa-2 cells. The total levels of Akt were not significantly affected (Fig. 6e). Immunofluorescence staining was used to detect the expression and localization of p-Akt-S473 and to further verify the results presented above. PDAC cells with LAMB3 


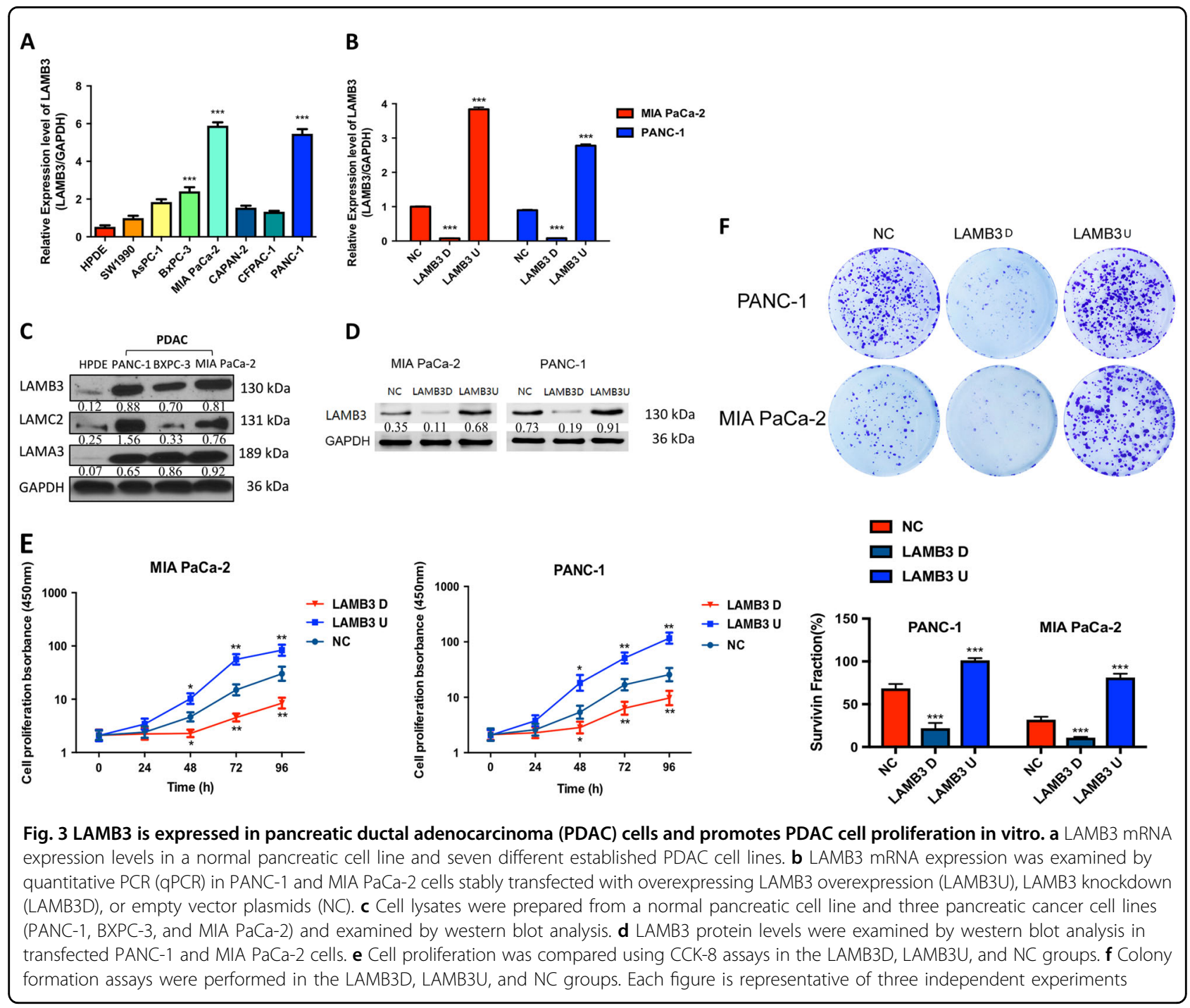

knockdown had decreased p-Akt-S473 levels, which correlated with an EMT phenotype, while the opposite results were observed in the LAMB3 overexpression group (Fig. 7).

\section{LAMB3 promotes pancreatic cancer cell proliferation in vivo}

To determine whether LAMB3 plays a role in PDAC proliferation in vivo, a subcutaneous xenograft model of PANC-1 and MIA PaCa- 2 cells in Balb/c nude mice was established. The LAMB3 knockdown group showed a slower increase in tumor volume and weight than did the control group (Fig. 8a, b). H\&E staining of serial tumor sections from the mouse xenograft model is shown in Fig. 8c, and LAMB3, Ki-67, and PCNA IHC was significantly reduced in the LAMB3 knockdown group compared with the $\mathrm{NC}$ group (Fig. 8d); the opposite effects were observed in the LAMB3 overexpression group.

\section{LAMB3 promotes pancreatic cancer cell invasion and migration in vivo}

A subcutaneous xenograft model and a model in which intrasplenically injected human PDAC cells induce hepatic metastasis were generated. In the xenograft model, mice were monitored and weighed weekly and then euthanized in the sixth week. There was no significant difference in tumor growth and weight in the NC, LAMB3 overexpression, and LAMB3 knockdown groups. H\&E staining of whole-xenograft serial sections indicated that mice injected with LAMB3-overexpressing cells had more metastatic foci than did control mice (Fig. 9a). LAMB3 IHC staining was significantly reduced in the LAMB3 knockdown group compared with the control group (Fig. 9b). Survival curves and gross pathologic analyses showed that LAMB3 knockdown decreased the number of liver metastatic nodules and prolonged the overall survival compared with the NC, while LAMB3 overexpression had the opposite effects (Fig. 9c, d). 


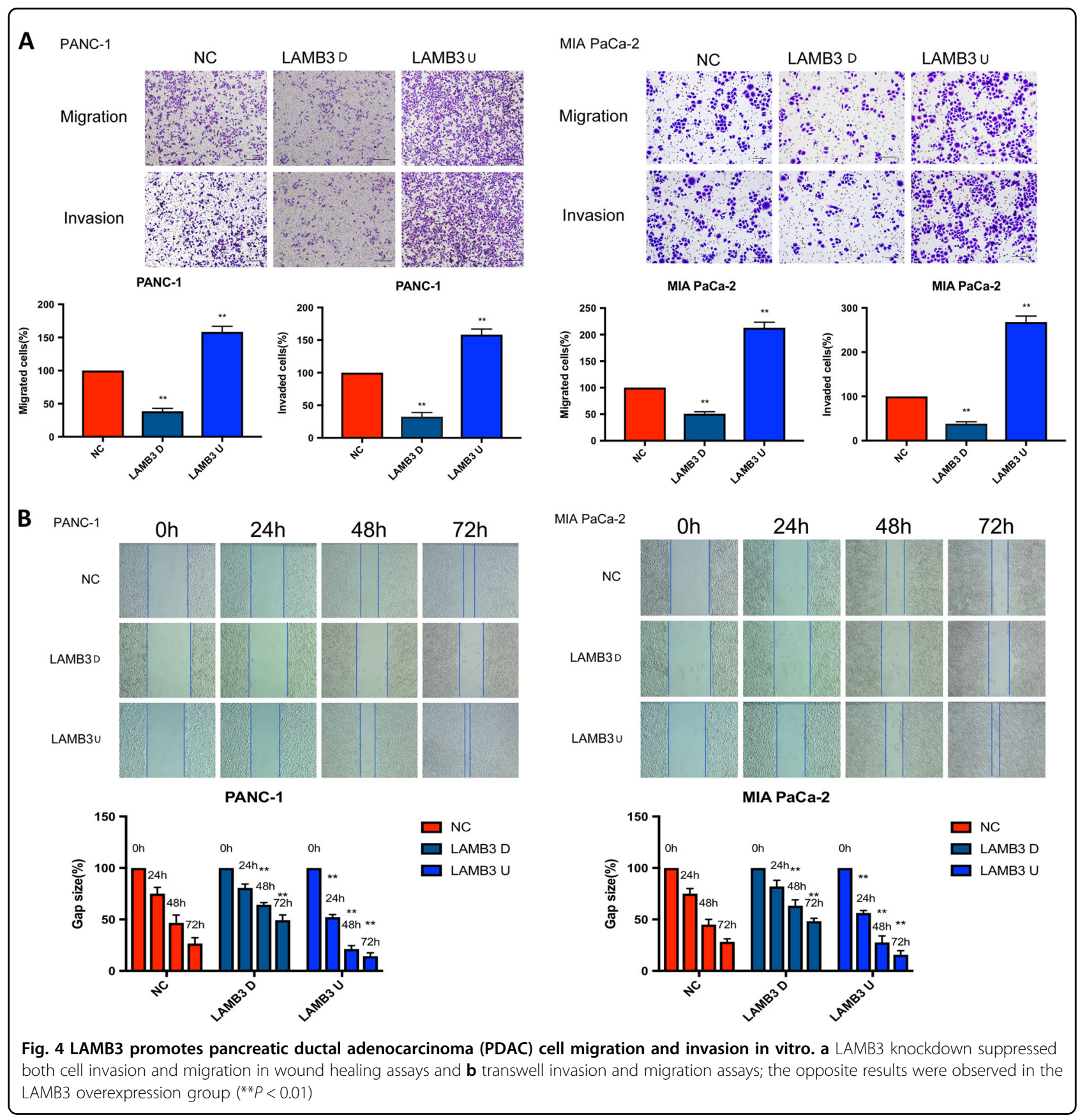

\section{Discussion}

Many recent studies have indicated that adhesion and extracellular matrix proteins contribute to the progression of a variety of solid tumors, including pancreatic cancer $^{20-22}$. LM-332, which is encoded by LAMA3, LAMB3, and LAMC2, is related to tumor invasiveness in various types of malignant tumors ${ }^{23-25}$. LAMB3 is a potential biomarker of cancer invasion and metastasis that is involved in the focal adhesion pathway, but a role for LAMB3 in pancreatic cancer has not been investigated previously $^{26,27}$.
In this study, we first evaluated LAMB3 expression in human PDAC and found it to be significantly higher in all PDAC tissues than in adjacent normal tissues. Clinical data from our retrospective study and the GSE and TCGA datasets also showed that LAMB3 overexpression was significantly associated with tumorigenesis and TNM stage in PDAC (Supplementary Table 1). In addition, we selected PANC-1 and MIA PaCa-2 cells (higher LAMB3 mRNA and protein expression) to conduct the following experiments. LAMB3 knockdown by short hairpin RNA (shRNA) had a significant effect on the proliferation of 


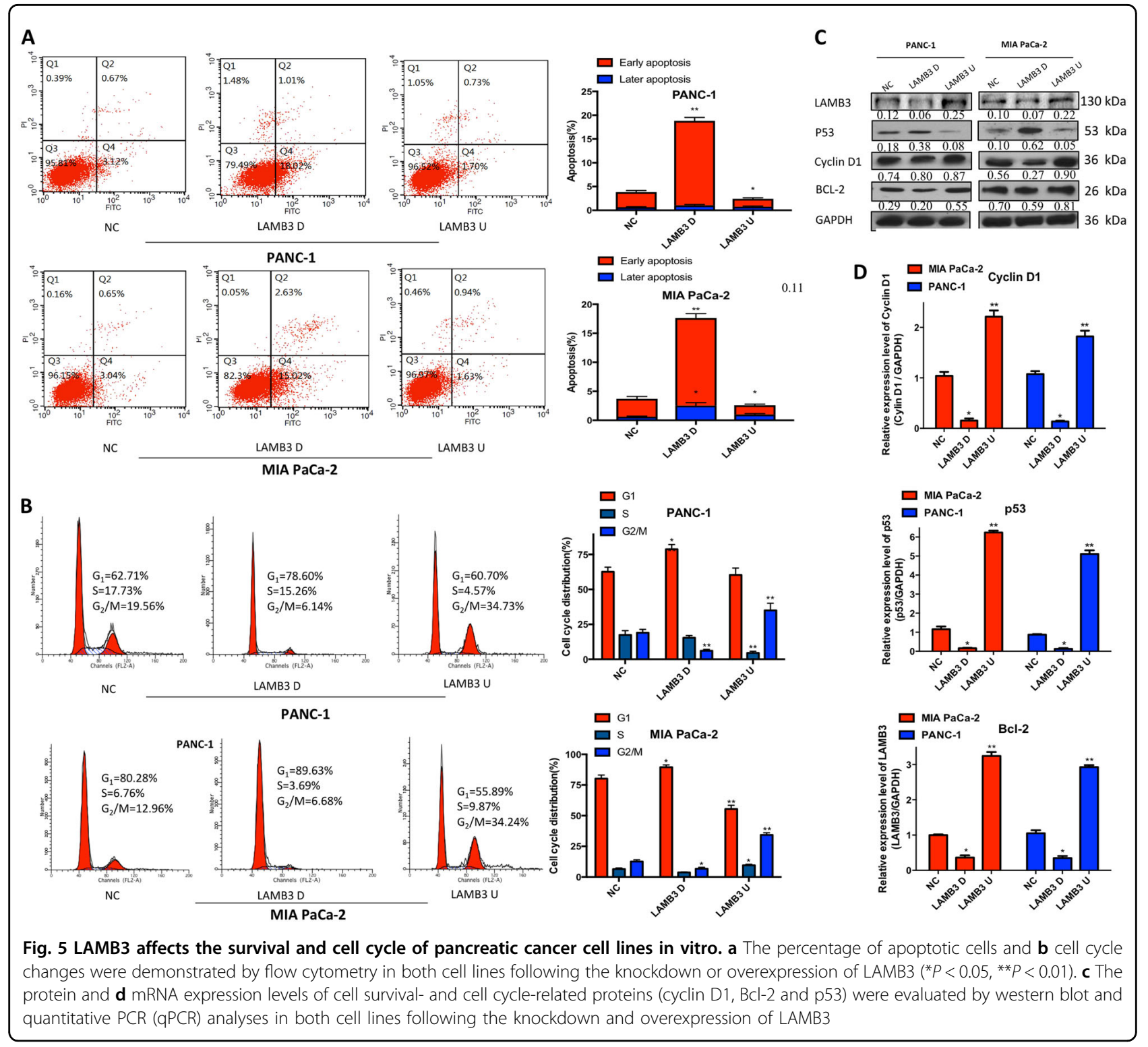

these two cell lines, as shown by CCK-8 and colony formation assays. In addition, the invasion and migration of PANC-1 and MIA PaCa-2 cells in transwell and wound healing assays were considerably reduced by LAMB3 knockdown, while LAMB3 overexpression had the opposite effects.

EMT is a process by which epithelial cells lose their polarity and adhesion after undergoing molecular reprogramming and phenotypic changes, and gain migratory and invasive properties to become mesenchymal stem cells $^{28-30}$. Our GSE analysis showed that LAMB3 overexpression was significantly related to cell adhesion in PDAC. LAMB3 knockdown by shRNA significantly reduced p-Akt-S473 levels without altering total Akt protein levels, indicating that LAMB3 could promote tumor invasion by activating the Akt signaling pathway in
PDAC. Increased Akt activity and Snail stabilization are essential for EMT. As a result, the expression of some proteins downstream of the Akt pathway, such as p53, BCL-2, and cyclin D1, which are involved in cell survival, antiapoptosis signaling and cell cycle arrest, respectively, were also influenced. MMPs are considered critical mediators of cellular events, including cell proliferation, apoptosis, invasion, migration, and morphological changes $^{31-33}$. Akt activation has been shown to induce EMT and MMP9 activity and to promote tumor invasiveness and metastasis ${ }^{34,35}$. Our analysis confirmed that LAMB3 regulates the EMT-related proteins E-cadherin, N-cadherin, Snail, Slug, vimentin, MMP2, and MMP9 via the activation of Akt signaling.

Our GSE analysis also showed that LAMB3 overexpression was positively correlated with the regulation of 


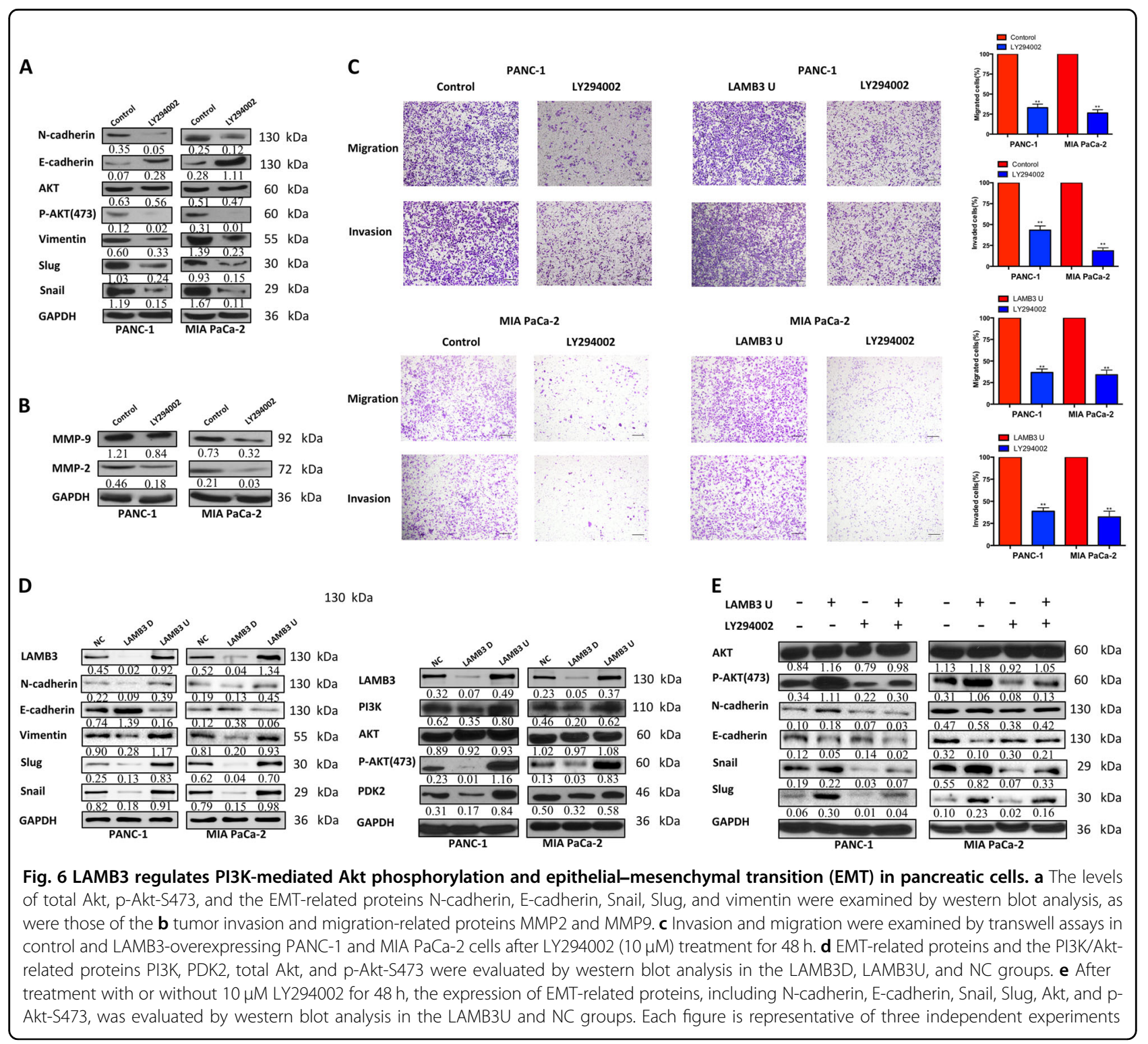

the phosphatidylinositol signaling system in PDAC. The PI3K/Akt signaling pathway is one of the best studied pathways in the phosphatidylinositol signaling system, which plays a crucial role in regulating normal cellular processes involved in tumorigenesis, cell growth, proliferation, metabolism, motility, survival, and apoptosis.

Aberrant activation of the PI3K/Akt signaling pathway promotes the survival and proliferation of tumor cells in many human cancers ${ }^{36-38}$. Akt is partially activated by T308 phosphorylation by PDK1. Full activation requires S473 phosphorylation, which can be catalyzed by multiple proteins, including $\mathrm{PDK} 2^{39,40}$. To investigate the upstream component that mediates Akt regulation by LAMB3, we focused on PI3K and PDK2. In our study, the PI3K/Akt pathway inhibitor LY294002 also significantly decreased the levels of p-Akt-S473, N-cadherin, Snail, and
Slug in both the NC and LAMB3-overexpressing groups, whereas E-cadherin levels were significantly increased in only LAMB3-overexpressing MIA PaCa- 2 cells. Total Akt levels were not significantly affected. These results further indicated that LAMB3-mediated invasion and migration may occur via the PI3K/Akt signaling pathway. Finally, we have shown that LAMB3 regulates the invasive and metastatic potential of PDAC in vivo by mouse tumor xenograft and liver metastasis models.

In summary, we demonstrated that LAMB3 promotes PDAC cell invasion and migration by activating the PI3K/ Akt signaling pathway in vitro and in vivo. Our present results showed that LAMB3 is upregulated in PDAC tissues, and high LAMB3 expression is linked to processes involved in tumorigenesis, such as apoptosis, cell cycle, proliferation, migration and invasion, by activating the 


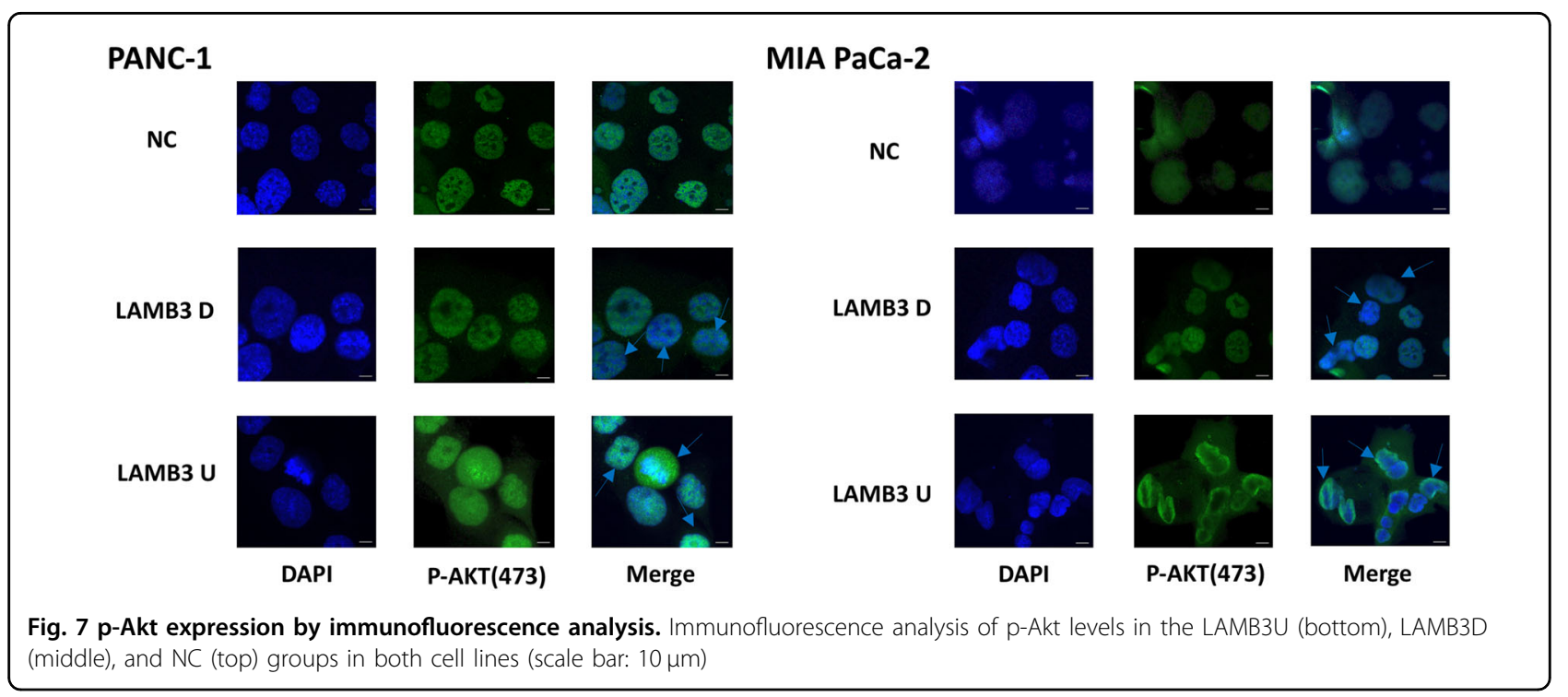

PI3K/Akt signaling pathway in PDAC. To our knowledge, this may be the first study to reveal that LAMB3 is related to the occurrence and development of PDAC and to elucidate the relevant molecular mechanism of LAMB3 in PDAC. At present, many inhibitors of the PI3K/Akt signaling pathway have been developed, such as LY294002, PX-866, BKM120, SAR245408, XL147, and GDC$0941^{41,42}$. Our study may provide a potential target for pharmaceutical development and novel therapeutic strategies for the use of these PI3K/Akt pathway inhibitors to control PDAC invasiveness and metastasis in the future. Furthermore, we found that PDAC patients with high LAMB3 expression presented with a relatively more advanced TNM stage. Thus, high LAMB3 expression may be an independent prognostic factor in patients with resected pancreatic carcinoma and a new clinical indicator for choosing the appropriate individualized treatment method ${ }^{43}$.

\section{Materials and methods}

\section{Cell culture and reagents}

The human pancreatic cancer cell lines PANC-1 and MIA PaCa-2 were purchased from ATCC and grown in Dulbecco's Modified Eagle Medium (DMEM) supplemented with $10 \%$ heat-inactivated fetal bovine serum (FBS; Gibco, CA, USA). All cells were maintained in a standard humidified incubator $\left(37^{\circ} \mathrm{C}, 5 \% \mathrm{CO}_{2}\right)$. LY294002 was purchased from Cell Signaling Technology, Inc. (MA, USA).

\section{Cell proliferation assay}

PANC- 1 and MIA PaCa- 2 cells $\left(3 \times 10^{3}\right.$ cells/well $)$ were seeded in 96-well plates overnight. All cells were transfected with small interfering RNA (siRNA) on the next day. CCK-8 solution (Abcam, MA, USA) was added to each well. After $2 \mathrm{~h}$ of incubation, the plates were shaken, and the absorbance was measured using a SpectraMax M5 plate reader (Molecular Devices, Sunnyvale, CA, USA) at $450 \mathrm{~nm}$ (OD450). The same procedure was repeated every $24 \mathrm{~h}$ until the last plate had been assayed. All assays were repeated in triplicate (Fig. 10).

\section{Wound healing assay}

PANC- 1 and MIA PaCa- 2 cells $\left(1 \times 10^{6}\right.$ cells/well $)$ were cultured in 6-well plates. A wound was generated in confluent cell monolayers by standardized wound scratching using a sterile $200 \mu \mathrm{l}$ pipette tip, and the cells were then incubated in culture medium with $1 \% \mathrm{FBS}$ and $5 \mathrm{ng} / \mathrm{ml}$ TGF- $\beta$. Cell migration into the wound area and recovery of the monolayer were monitored $(0-72 \mathrm{~h})$ by a phase contrast microscope and digitally photographed (Nikon Diaphot 300; Nikon, Tokyo, Japan).

\section{Cell invasion and migration assays}

Transwell membranes (Corning, NY, USA) were coated with Matrigel for $6 \mathrm{~h}$ for invasion assays and used without Matrigel for migration assays. PANC- 1 and MIA PaCa-2 cells $\left(0.05 \times 10^{6}\right)$ in serum-free medium were seeded into the upper chambers, and $600 \mu \mathrm{l}$ of medium supplemented with $10 \%$ FBS was added to the lower chambers. After incubation for $24 \mathrm{~h}$, the cells adhering to the upper surface of the membrane were removed. Meanwhile, the invaded or migrated cells, which adhered to the lower surface, were stained with $0.1 \%$ crystal violet and measured by optical microscopy.

\section{Real-time quantitative polymerase chain reaction (qPCR)}

Total RNA was extracted from PANC-1 and MIA PaCa2 cells using TRIzol reagent (Invitrogen, CA, USA), 


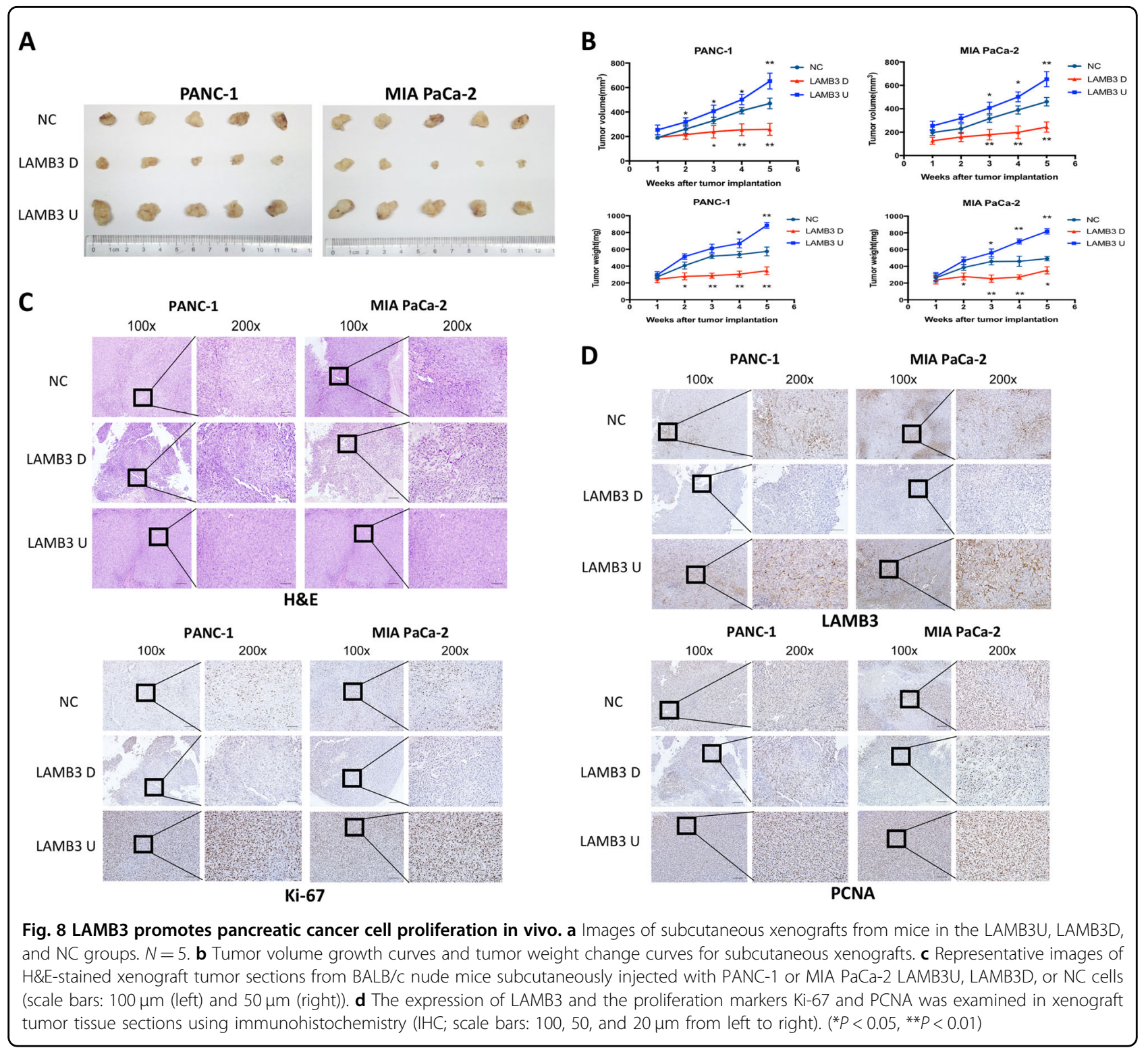

reverse transcribed and amplified using primers for LAMB3 and glyceraldehyde 3-phosphate dehydrogenase (GAPDH). The RT-PCR primer sequences (Invitrogen, CA, USA) were LAMB3 (forward) 5'-CCAAAGGTGC GACTGCAATG-3' and (reverse) $5^{\prime}$-AGTTCTTGCCTT CGGTGTGG-3' and GAPDH (forward) $5^{\prime}$-ACAACTTT GGTATCGTGGAAGG-3' and (reverse) 5'-GCCATCA CGCCACAGTTTC-3'.

\section{Colony formation assay}

Cells were seeded at an initial density of 500 cells/well and cultured in six-well plates. After 2 weeks of cultivation, the cells were fixed with $4 \%$ paraformaldehyde and stained with $0.1 \%$ crystal violet (Sigma, MO, USA). Colonies containing over 50 cells were counted using a light microscope.

\section{Immunofluorescence}

Cells $\left(0.02 \times 10^{6}\right)$ were seeded on glass cover slips (Thermo Fisher Scientific, MA, USA) and cultured in a standard humidified incubator $\left(37^{\circ} \mathrm{C}, 5 \% \mathrm{CO}_{2}\right)$. The cells were fixed with $500 \mu \mathrm{l}$ of $4 \%$ paraformaldehyde for $10 \mathrm{~min}$, permeabilized in $0.2 \%$ Triton X-100 for 5 min and blocked with $5 \%$ BSA for $30 \mathrm{~min}$ at room temperature. Primary antibodies against p-Akt-S473 (Cell Signaling Technology, MA, USA) and LAMB3 (Abcam, MA, USA) were added to the slides at a 1:100 dilution in 5\% BSA, and the slides were incubated overnight in a moist box in the cold room; Phosphate-Buffered Saline (PBS) was added to the NC slides. The next morning, Cy3- and Cy5-labeled secondary antibodies (Thermo Fisher Scientific, MA, USA) were added at a 1:200 dilution in PBS, and the slides were incubated for $1 \mathrm{~h}$ at room temperature in the dark. All 


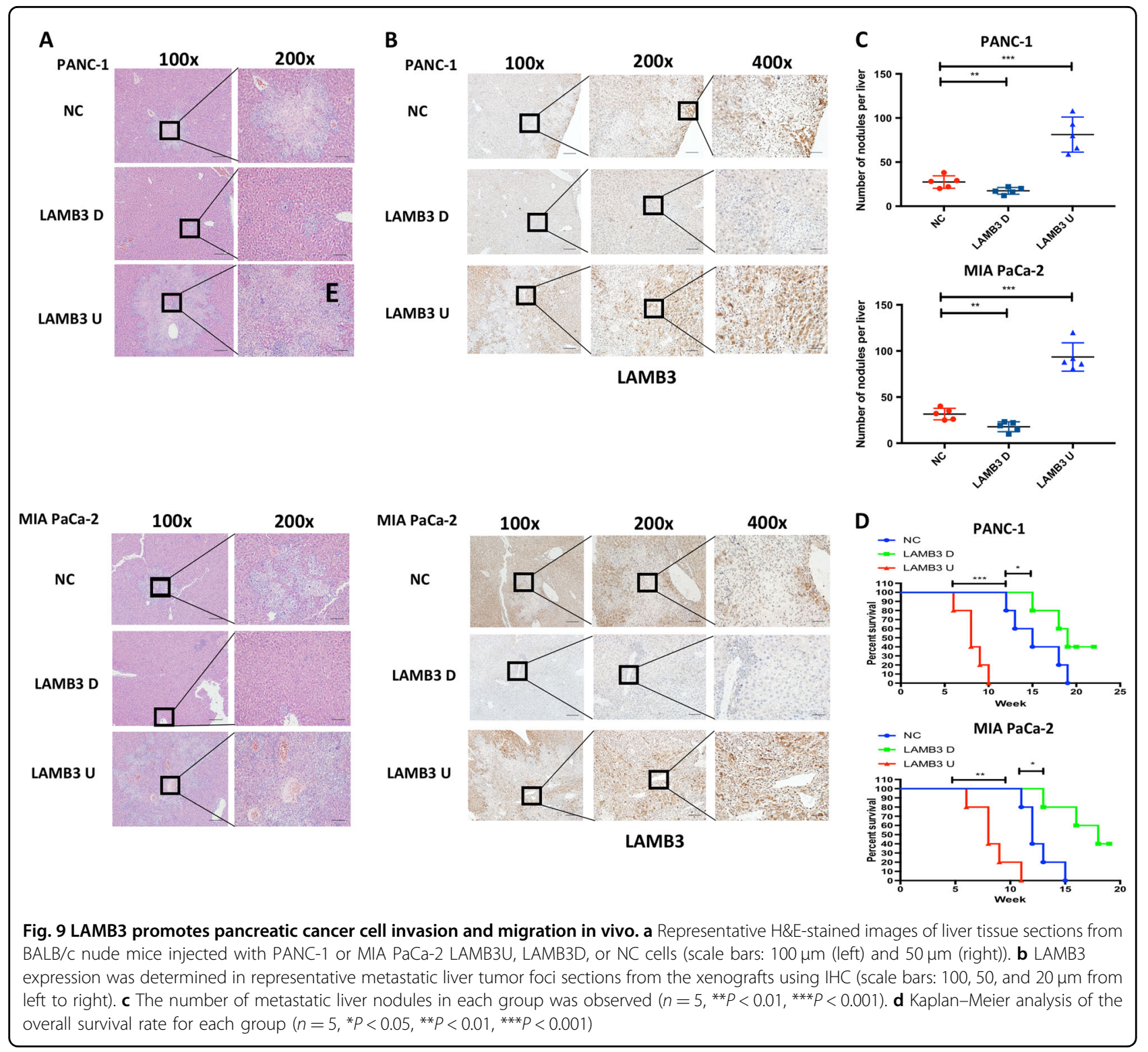

washes were performed with $1 \times$ PBS. The slides were mounted in antifade solution containing DAPI (Thermo Fisher Scientific, MA, USA). Images were taken with a Leica TCS SP8 confocal microscope (Leica, IL, USA).

\section{Western blot analysis}

Cells were lysed in lysis buffer containing $50 \mathrm{mM}$ HEPES, $\mathrm{pH} 8.0,10 \%$ glycerol, $1 \%$ Triton X-100, $150 \mathrm{mM} \mathrm{NaCl}$, $1 \mathrm{mM}$ EDTA, $1.5 \mathrm{mM} \mathrm{MgCl}, 100 \mathrm{mM} \mathrm{NaF}, 10 \mathrm{mM}$ $\mathrm{Na}_{4} \mathrm{P}_{2} \mathrm{O}_{7} \cdot 10 \mathrm{H}_{2} \mathrm{O}$, and a protease inhibitor cocktail (Roche Applied Science, Mannheim, Germany). Frozen tissue samples stored in liquid nitrogen were cut into pieces with scissors. Each sample was homogenized in lysis buffer at a ratio of 1:20. After centrifugation at 14,000 rpm for $20 \mathrm{~min}$, the supernatant was collected. A BCA Protein Assay kit
(Thermo Scientific, IL, USA) was used to measure total protein concentration. Aliquots $(50 \mu \mathrm{g})$ of total cellular protein were resolved by sodium dodecyl sulfate polyacrylamide gel electrophoresis (6-15\%) and electrotransferred onto Polyvinylidene Fluoride (PVDF) membranes, which were blocked with $5 \%$ skim milk at room temperature for $1 \mathrm{~h}$. The membranes were then incubated with primary antibodies against the following proteins for western blot analysis: LAMB3, E-cadherin, and N-cadherin (1:1000; Abcam, MA, USA); phosphoAkt-S473, total Akt, vimentin, Slug, Snail, and $\beta$-actin (1:1,000; Cell Signaling Technology, MA, USA); and LAMA3 and LAMC2 (1:1000; Santa Cruz Biotechnology, Santa Cruz, CA, USA). Following incubation with the corresponding secondary antibodies (1:5000; GE 


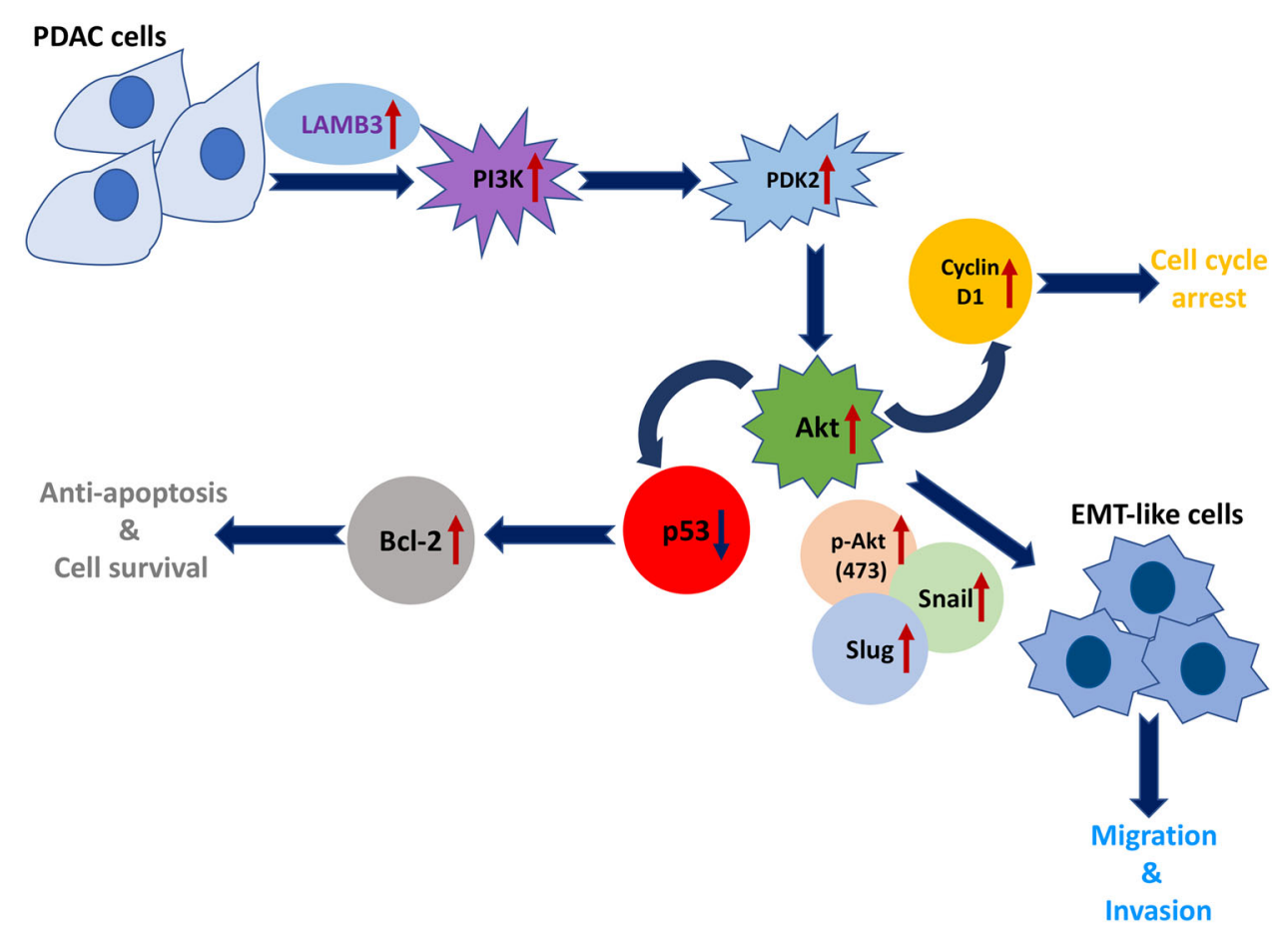

Fig. 10 The proposed mechanism of LAMB3-mediated tumor antiapoptotic signaling, invasion and metastasis through the PI3K/Akt axis in patients with pancreatic ductal adenocarcinoma (PDAC)

Healthcare, Buckinghamshire, UK), immune reactive bands were visualized by enhanced chemiluminescence (ECL) detection.

\section{IHC staining and TMAs}

TMAs were created by Shanghai Outdo Biotechnology Co., Ltd. (Shanghai, China). IHC staining with antibodies against LAMB3, Ki-67, and PCNA (Abcam, MA, USA) was performed to detect protein expression levels following standard operating procedures. The positive staining scores were calculated by multiplying the percentage positive $(0,<5 \%$ of cells; $1,5-25 \% ; 2,26-50 \% ; 3,51-75 \%$; and $4,76-100 \%)$ by the staining intensity score $(0$, no coloration; 1 , pale yellow; 2 , yellow; and 3 , dark brown) and were classified as follows: negative $(0,-)$; weakly positive $(1-3,+)$; moderately positive $(4-8,++)$; and strongly positive $(>8,+++)$. We divided all patients into two groups $(-/+$, low expression; and $++/+++$, high expression) and performed survival analyses.

\section{Plasmid and lentivirus production}

We used the lentiviral vector pLKO.1-puro-CMV-TurboGFP (VT8114, Youbio, Changsha, China) to knock down LAMB3 expression (LAMB3D). The sequence of the 21-nucleotide shRNA targeting LAMB3 was GAAGC TTCAATGGTCTCCTTA. LAMB3 was overexpressed (LAMB3U) using pCDH-CMV-MCS-EF1-puro (VT1480,
Youbio, Changsha, China). Knockdown or overexpression lentivirus particles were generated by cotransfecting lentivirus constructs with pMD2.G (plasmid 12259, Addgene, Cambridge, MA, USA) and psPAX2 (plasmid 12260, Addgene, Cambridge, MA, USA) at a 4:3:1 ratio into HEK-293T cells. The pCDH-CMV-MCS-EF1-puro empty vector was used as a NC.

\section{Tumor xenograft model}

Female 6-week-old BALB/c nude mice were provided by the Experimental Animal Study Center of Hubei Province. All mice were bred under specific pathogen-free conditions in the Experimental Animal Center of the Wuhan Institute of Virology, CAS. Female age-matched mice were used in all experiments. A total of $1 \times 10^{7}$ transfected cells were subcutaneously injected into the left armpit of the mice ( $n=5$ per group). Mouse weight and tumor diameter were measured every week. Mice were sacrificed 6 weeks after the initiation of treatment, and the tumor weight and volume were evaluated.

\section{Pancreatic cancer liver metastasis model}

Human pancreatic cancer cells $\left(5 \times 10^{6}\right)$ were resuspended in serum-free medium and injected into the involucrum of the spleen of female 8 -week-old BALB/c nude mice ( $n=5$ per group). Splenectomy was performed $30 \mathrm{~min}$ later. Mice were weighed once a week and 
sacrificed when cachexia was observed or if the mice exhibited $>15 \%$ weight loss. Mouse livers were subsequently evaluated both macroscopically and microscopically.

\section{Statistical analysis}

All experiments were performed in triplicate and were independently conducted three times. GraphPad Prism 7.0 (GraphPad Software, USA) was used for the statistical analyses. Data are presented as the mean \pm SD. Statistical significance was determined using Student's $t$-test for the two groups and one-way ANOVA for multiple groups. Significant differences between two mean values were estimated using Student's $t$-test. A $P$-value $<0.05$ was considered to indicate statistical significance.

\section{Acknowledgements}

This work is supported by 1. Clinical Medical Research Center of Hepatobiliary Surgery of Guizhou Province, Platform Talent of Scientific Collaboration of Guizhou Province (No. [2017] 5404); 2. High-Level Innovative Talent Training Program "Ten" Level Talents of Guizhou Province, Platform Talent of Scientific Collaboration of Guizhou Province (No. [2016] 5647); 3. Brainstorm Project on Social Development by the Department of Science and Technology of Guizhou Province (No. SY [2015] 3047); 4. Youth Science and Technology Talent Growth Project by the Department of Education of Guizhou Province (No. KY [2016] 142); 5. Tutor Studio of Sun Chengyi's "Diagnosis and Treatment of Hepatobiliary, Pancreatic and Splenic Diseases", Teaching and Research Collaboration of Guizhou Province (No. GZS [2016] 09); 6. Department of Science and Technology of Guizhou Province, Hepatobiliary Surgery, Station of Academician Workstation of Guiyang Medical College, Academician Station of Scientific Collaboration of Guizhou Province (No. [2015] 4013); 7. United Fund of the Department of Science and Technology of Guizhou Province and Affiliated Hospital of Guizhou Medical University, Scientific Collaboration of Guizhou Province (No. LH [2016] 7229); 8. Fund of The Fourth Talent Base of Guizhou Province, Special Collaboration of Guizhou Province (No. [2012]94).

\section{Author details}

${ }_{1}^{1}$ Guizhou Medical University, Guiyang, Guizhou, China. ${ }^{2}$ Department of Hepatobiliary Surgery, The Affiliated Hospital of Guizhou Medical University, Guiyang, Guizhou, China. ${ }^{3}$ Key Laboratory of Hepatobiliary-Pancreas-Spleen Surgery of Guizhou Medical University, Guiyang, Guizhou, China. ${ }^{4}$ Ontario Cancer Institute, Princess Margaret Cancer Centre, University of Toronto, Toronto, ON, Canada

\section{Conflict of interest}

The authors declare that they have no conflict of interest.

\section{Publisher's note}

Springer Nature remains neutral with regard to jurisdictional claims in published maps and institutional affiliations.

Supplementary Information accompanies this paper at (https://doi.org/ 10.1038/s41419-019-1320-z).

Received: 12 September 2018 Revised: 8 December 2018 Accepted: 17 December 2018

Published online: 08 March 2019

\section{References}

1. Yu, C., Chen, S. Y., Guo, Y. T. \& Sun, C. Y. Oncogenic TRIM31 confers gemcitabine resistance in pancreatic cancer via activating the NF-KB signaling pathway. Theranostics 8, 3224-3236 (2018).
2. Zhou, X., Guo, X. J., Xie, C. C. \& Jiang, J. X.HIF-3a promotes metastatic phenotypes in pancreatic cancer by transcriptional regulation of the RhoC-ROCK1 signaling pathway. Mol. Cancer Res. 16, 124-134 (2018).

3. Wang, J., Guo, X. J., Xie, C. C. \& Jiang, J. X. KIF15 promotes pancreatic cancer proliferation via the MEK-ERK signalling pathway. Br. J. Cancer 117, 245-255 (2017).

4. Chen, Y. W. et al. SMAD4 loss triggers the phenotypic changes of pancreatic ductal adenocarcinoma cells. BMC Cancer 14, 181 (2014).

5. Wang, Y. et al. Oncogenic functions of Gli1 in pancreatic adenocarcinoma are supported by Its PRMT1-mediated methylation. Cancer Res. 76, 7049-7058 (2016).

6. Chandrashekar, D. S. et al. UALCAN: A portal for facilitating tumor subgroup gene expression and survival analyses. Neoplasia 19, 649-658 (2017).

7. Jung, S. N. et al. LAMB3 mediates metastatic tumor behavior in papillary thyroid cancer by regulating c-MET/Akt signals. Sci. Rep. 8, 2718 (2018).

8. Benati, D. et al. CRISPR/Cas9-mediated in situ correction of LAMB3 gene in keratinocytes derived from a junctional epidermolysis bullosa patient. Mol. Ther. pii: S1525-0016(18)30371-X. https:/doi.org/10.1016/j.ymthe(2018).

9. Wang, Y. et al. Upregulated LAMB3 increases proliferation and metastasis in. thyroid cancer. Onco. Targets Ther. 11, 37-46 (2017).

10. Hou, J., Wang, L. \& Wu, D. The root of Actinidia chinensis inhibits hepatocellular carcinomas cells through LAMB3. Cell Biol. Toxicol. 34, 321-332 (2018).

11. Laroussi, N. et al. Identification of a novel mutation of LAMB3 gene in a Lybian patient with hereditary epidermolysis bullosa by whole exome sequencing. Ann. Dermatol. 29, 243-246 (2017).

12. Ersahin, T., Tuncbag, N. \& Cetin-Atalay, R. The PI3K/AKT/mTOR interactive pathway. Mol. Biosyst. 11, 1946-1954 (2015).

13. Hemmings, B. A. \& Restuccia, D. F. PI3K-PKB/Akt pathway. Cold Spring Harb. Perspect. Biol. 4, a011189 (2012).

14. Li, X. et al. Synergistic antitumor activity of withaferin A combined with oxaliplatin triggers reactive oxygen species-mediated inactivation of the PI3K/AKT pathway in human pancreatic cancer cells. Cancer Lett. 357, 219-230 (2015).

15. Huang, M. et al. Suppresses growth of human pancreatic cancer xenografts, and. pancreatic cancer cells isolated from KrasG12D mice by inhibiting Akt and Sonic hedgehog pathways. PLoS ONE 9, e92161 (2014).

16. Xu, J., Gong, L., Qian, Z., Song, G. \& Liu, J. ERBB4 promotes the proliferation of gastric cancer cells via the PI3K/Akt signaling pathway. Oncol. Rep. 39, 2892-2898 (2018)

17. Sharma, N. et al. PI3K/AKT/mTOR and sonic hedgehog pathways cooperate. together to inhibit human pancreatic cancer stem cell characteristics and tumor growth. Oncotarget 6, 32039-32060 (2015).

18. Jiang, $\mathrm{H}$. et al. Concurrent HER or PI3K inhibition potentiates the anti-tumor effect of ERK. Inhibitor ulixertinib in preclinical pancreatic cancer models. Mol. Cancer Ther. pii: molcanther.1142.2017. https://doi.org/10.1158/1535-7163. MCT-17-1142 (2018).

19. Baer, R., Cintas, C., Therville, N. \& Guillermet, G. J. Implication of PI3K/Akt pathway. in pancreatic cancer: When PI3K isoforms matter? Adv. Biol. Regul. 59, 19-35 (2015).

20. Meng, Q. et al. Abrogation of glutathione peroxidase-1 drives EMT and chemoresistance in pancreatic cancer by activating ROS-mediated Akt/GSK3B/ Snail signaling. Oncogene. https://doi.org/10.1038/s41388-018-0392-z (2018).

21. Chen, S. T. et al. Silencing of MUC20 suppresses the malignant character of pancreatic ductal adenocarcinoma cells through inhibition of the HGF/MET pathway. Oncogene. https://doi.org/10.1038/s41388-018-0403-0 (2018).

22. Tan, J. et al. Par-4 downregulation confers cisplatin resistance in pancreatic cancer cells via PI3K/Akt pathway-dependent EMT. Toxicol. Lett. 224, 7-15 (2014).

23. Gostyńska, K. B. et al. Carriers with functional null mutations in LAMA3 have localized enamel abnormalities due to haploinsufficiency. Eur. J. Hum. Genet. 25, 94-99 (2016).

24. Zhou, Y. et al. miR-1298 Inhibits Mutant KRAS-Driven Tumor Growth by Repressing FAK and LAMB3. Cancer Res. 76, 5777-5787 (2016).

25. Fuentes, l. et al. Molecular epidemiology of junctional epidermolysis bullosa: discovery of novel and frequent LAMB3 mutations in Chilean patients with diagnostic significance. Br. J. Dermatol. 176, 1090-1092 (2017).

26. Mayer, B. et al. A unique LAMB3 splice-site mutation with founder effect from the Balkans causes lethal epidermolysis bullosa in several European countries. Br. J. Dermatol.175, 721-727 (2016).

27. Stemmler, S. et al. Association of variation in the LAMA3 gene, encoding the alpha-chain of laminin 5, with atopic dermatitis in a German case-control cohort. BMC Dermatol. 14, 17 (2014). 
28. Zhao, $H$. et al. MiR-543 promotes migration, invasion and epithelialmesenchymal transition of esophageal cancer cells by targeting phospholipase A2 group IVA. Cell. Physiol. Biochem. 48, 1595-1604 (2018).

29. Zhao, $\mathrm{H}$. et al. Activation of glucagon-like peptide-1 receptor inhibits tumourigenicity and metastasis of human pancreatic cancer cells via PI3K/Akt pathway. Diabetes Obes. Metab. 16, 850-860 (2014).

30. Xu, Q. et al. a-Mangostin suppresses the viability and epithelial-mesenchymal transition of pancreatic cancer cells by downregulating the PI3K/Akt pathway. Biomed. Res. Int. 20, 546353 (2014).

31. Liang, J. et al. CDK8 selectively promotes the growth of colon cancer metastases in the liver by regulating gene expression of TIMP3 and matrix metalloprotein ases. pii: canres.1583.2018. https://doi.org/10.1158/0008-5472. CAN-18-1583 (2018).

32. Park, J. Y. et al. Eupatilin inhibits angiogenesis-mediated human hepatocellular metastasis by reducing MMP-2 and VEGF signaling. pii: S0960 -894X(18) 30711-X. https://doi.org/10.1016/j.bmcl.2018.08.034 (2018).

33. Itoh, Y. Membrane-type matrix metalloproteinases: Their functions and regulations. Matrix Biol. 46, 207-223 (2015).

34. He, L. et al. Imbalance of the reciprocally inhibitory loop between the ubiquitin-specific protease USP43 and EGFR/PI3KJAKT drives breast carcinogenesis. Cell Res. https://doi.org/10.1038/s41422-018-0079-6 (2018).

35. Mao, Y. et al. Combination of PI3K/Akt pathway inhibition and Plk1 depletion can enhance chemosensitivity to gemcitabine in pancreatic carcinoma. Transl. Oncol. 11, 852-863 (2018)
36. Bai, R. et al. The effect of PI3K inhibitor LY294002 and gemcitabine hydrochloride combined. with ionizing radiation on the formation of vasculogenic mimicry of Panc-1 cells in vitro and in vivo. Neoplasma 63, 80-92 (2016).

37. Zhao, Z., Li, C., Xi, H., Gao, Y. \& Xu, D. Curcumin induces apoptosis in pancreatic cancer cells through the induction of forkhead box $\mathrm{O} 1$ and inhibition of the PI3K/Akt pathway. Mol. Med. Rep. 12, 5415-5422 (2015).

38. Liu, S., Wang, X. J., Liu, Y. \& Cui, Y. F. PI3K AKT/mTOR signaling is involved in (-)-epigallocatechin-3-gallate-induced apoptosis of human pancreatic carcinoma cells. Am. J. Chin. Med. 41, 629-642 (2013).

39. Wang, S. et al. Girdin regulates the proliferation and apoptosis of pancreatic cancer cells via the PI3KJAkt signalling pathway. Oncol. Rep. 40, 599-608 (2018).

40. Pan, Y. Z. et al. POU5F1B promotes hepatocellular carcinoma proliferation by activating AKT. Biomed. Pharmacother. 100, 374-380 (2018).

41. Akintunde, A., Avvaru, P., Muhammad, F., Song, Y. P. \& Liu, D. L. Phosphatidylinositol 3-kinase (PI3K) inhibitors as cancer therapeutics. J. Hematol. Oncol. 6, 88 (2013).

42. Jennifer, R. B. et al. Phase I trial of the Pan-PI3K inhibitor pilaralisib (SAR245408/ XL147) in patients with chronic lymphocytic leukemia (CLL) or relapsed/ refractory lymphoma. Clin. Cancer Res. 21, 3160-3169 (2015).

43. Van, R. S. et al. International validation of the eighth edition of the American Joint Committee on Cancer (AJCC) TNM staging system in patients with resected pancreatic cancer. JAMA Surg. https://doi.org/ 10.1001/jamasurg.2018.3617 (2018) 